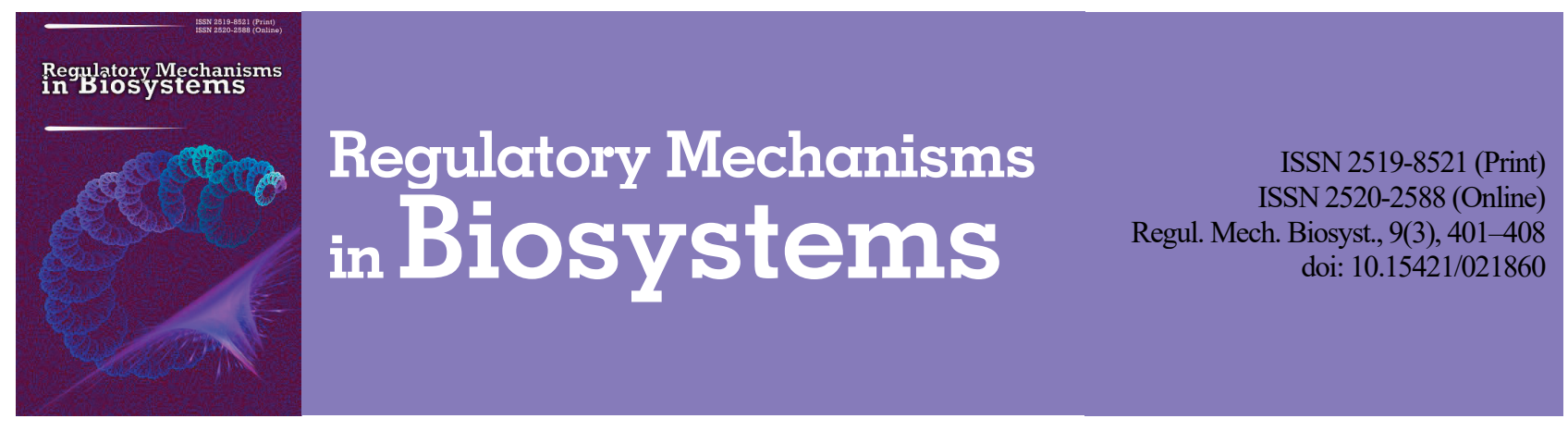

\title{
The characteristics, emergent properties and manner of spread in Ukraine of the Porcine Epidemic Diarrhea Virus
}

\author{
D. N. Masiuk*, V. S. Nedzvetsky*,**, A. I. Sosnitskiy*, A. V. Kokarev*, S. G. Koliada* \\ *Dnipro State Agrarian and Economic University, Dnipro, Ukraine \\ **Bingol University, Bingol, Turkey
}

Article info

Received 07.07.2018

Received in revised form 11.08 .2018

Accepted 13.08.2018

Dnipro State Agrarian and Economic University Sergei Efremov st., 25 , Dnipro, 49600, Ukraine. Tel.+38-056-236-17-14. E-mail:plppm@ua.fm

Bingol University

Selahaddin-i Eyyubi Mah Aydinlik Cad.,12000, Bingol,

Turkey.

E-mail:

nedzvetskyvictor@gmail.com

\begin{abstract}
Masiuk, D. N., Nedzvetsky, V. S., Sosnitskiy, A. I., Kokarev, A. V., \& Koliada, S. G. (2018). The characteristics, emergent properties and manner of spread in Ukraine of the Porcine Epidemic Diarrhea Virus. Regulatory Mechanisms in Biosystems, 9(3), 401-408. doi:10.15421/021860
\end{abstract}

Study of the emergent properties and paths of spreading of PEDV was carried out in a model experiment on newborn nonimmune piglets obtained from a PED virus-free pig-breeding enterprise. The piglets were kept in separate specialized containers, with a volume of $1.0 \mathrm{~m}^{3}$, with access only through the opening at the top of the containers. The experimental group of the animals was infected with PEDV isolate extracted on one of the pig farms from the central region of Ukraine. Infection was carried out orally in a dose of 1-10 genome equivalents of virions. The control piglets, which were situated in the same room as the infected animals, were not deliberately infected. The study of biological material from piglets was carried out using the methods of bacteriology, histology and RT-PCR. To confirm the capability of PEDV to spread through house flies, specimens of Musca domestica vicina Mcq. were caught in the building where the experiment was carried out. The washings from the surface of their bodies were collected with sterile saline. Individually, 28 specimens of flies were selected. They were divided into two parts and the amount of virus in the homogenate of the fly bodies in these groups was determined with an interval between measurements of 72 hours. Study of PEDV in the washings and in flies body homogenates were carried out using RT-PCR. It is established that the field strain PEDV, belonging to the North American grouping II of the second group of the PED virus strains, is an emergent highly pathogenic agent for nonimmune newborn piglets. In the model of piglets' infection it is established that the tested PEDV strain has a high virulence for newborn piglets, DCL is 1-10 virions and the incubation period is 18-26 hours. PED is acute with lethality to $100 \%$ within $68-$ 72 hours after infection. According to the results of RT-PCR in washing from the surface of the bodies of flies, it is established that one of the ways piglets are infected and the environment contaminated with the PED virus is the spread of the pathogen by the flies M. domestica vicina Mcq. This leads to the induction of the emergent form of PED in piglets. The presence of PEDV in the homogenate from bodies of $M$. domestica caught in the focus of infection and the absence of virus reproduction in their body confirms the role of the house fly in the mechanical spread of PEDV in the external environment.

Keywords: PEDV; piglets; pathways; emergence; DCL; flies; Musca domestica.

\section{Introduction}

The virus of epidemic diarrhea of pigs (Porcine epidemic diarrhea virus - PEDV) was recently identified as a new infection and is considered as the causative agent of an emergent nosological unit in infectology. Epidemic diarrhea of pigs (PED) was first recorded in Europe at 1971, in South-East Asia 1995, in the Russian Federation 2005 and in Ukraine 2014 (Song et al., 2005; Martelli et al., 2008; Puranaveja et al., 2009; Strizhakova, 2013). In a relatively short time, the infection has assumed a global scope and tends to stagnate (Gerber et al., 2014; Vlasova et al., 2014; Wang et al., 2014; Dastjerdi et al., 2015).

Pigs of all age groups are affected by PED, though the most susceptible to PEDV are newborn piglets up to 10 days of age. In newborn piglets there is practically no immunity, and this is the main cause of their high susceptibility to infection with PEDV. In the early postnatal period, piglets develop debilitating watery diarrhea, dehydration, metabolic acidosis, intoxication and exhaustion during the course of the disease, which inevitably leads to death. From birth to 5 days, deaths reach $100 \%$, from 6 to 10 days $-60 \%$, from 11 to 15 days $-30 \%$, further $3 \%$ or less. When an infectious agent of PEDV reaches a farm, suckling piglets fall sick in the first days of life and die within 2-4 days. Such a progressive development of the infectious process determines the ineffectiveness and uselessness of vaccinating piglets. Under the circumstances of the infection propagation, the specific immune-biological protection with colostral immunoglobulins can form lactogenic immunity only (Annamalai et al., 2015).

Nowadays highly effective and biosafe methods of specific immune-prophylaxis and therapy are still not developed. Existing veterinary medications do not ensure eradication of the pathogen and are not effective against the expansion of PEDV on susceptible animals, therefore the development of epizootic PED is a difficult process to control (Choudhury et al., 2016). Both the practically total death of neonatal pigs and the absence of an etiopathogenetic correction of the infectious process are characteristic features of PEDV infection. The uncontrolled spread of the causative agent in swine herds can lead to enormous economic losses in industrial pig production. Together these facts give the grounds for considering PED as an emergent and especially dangerous infection in pig breeding (Gong et al., 2017).

The main path of infection of animals with the PED causative agent is oral-fecal with the subsequent replication of the virions in epithelial cells of the intestine, which leads to their prompt necrosis, tissue destruction and desquamation. After a short incubation period that varies in the range of 3-6 days, pathognomonic clinical signs of the disease appear in the form of diarrhea syndrome, which is accompanied by 
watery diarrhea, vomiting, dehydration, increasing weakness, depresssion, exhaustion, intoxication and, as a rule, by death (Masiuk et al., 2017a). The excretion of PEDV with feces lasts 1-3 days, at the same time viral RNA is identified by molecular genetic methods within a month. After infection, a relatively short (up to 5 days) viremia period is usually detected. Viral antigens are detected in intestinal enterocytes as early as 12 hours after infection. Moreover, diagnosed concentration PEDV remains up to two weeks during the piglet infection (Lee, 2015).

The causative agent possesses unique biological properties that ensure the progressive growth of the infectious process (Boyko et al., 2009). The most important epizootic factors are high pathogenicity, extreme contagiousness, expressed enterotrophy and fatalities for newborn piglets, which together account for its emergent properties as a causative agent of a difficult to control infectious pathology (Kim \& Chae, 2003; Martelli et al., 2008; Carvajal et al., 2015; Dastjerdi et al., 2015; Goede et al., 2015; Diel et al., 2016).

Given the fact that the virus causes non-curable diarrheal syndrome in newborn piglets, which leads to their total death, lactogenic immunity could prove to be the main method of their protection. It is impossible to form effective immunity without high immunogenic vaccines for sows. Modern anti-viral chemicals do not have sufficient protective activity for arresting the epizootic process of PED (Strizhakova, 2013; Langel et al., 2016; Tun et al., 2016; Srijangwad et al., 2017). Because of all of the above factors, the biological properties of PEDV field strains and epizootic patterns of PED, especially the emergent infectious pathology of piglets, this virus infection is recognized as one of the most urgent problems in animal infectious diseases. Quantitative characteristics of the pathogenicity of field strains of the PEDV, the paths of dissemination of this virus in the environment and the pathogenesis of PED have been insufficiently studied. The results of a detailed study of PEDV biology are extremely important as a methodological basis for developing strategies and tactics for combating and preventing its infectious pathology (Arriba et al., 2002; Stevenson et al., 2013; Goede \& Morrison, 2016).

The aim of this study was to clarify the manner of the spread, the degree of pathogenicity and emergent properties of the PEDV field isolate in Ukraine in the model of native conditions of infection of noncolostrum, non-immune piglets.

\section{Material and methods}

The empirical component of the model experiment on the assessment of the emergent proprieties of PED infection was carried out in the vivarium of the Faculty of Veterinary Medicine of the Dnipro State Agrarian and Economic University (DSAEU). The laboratory research was carried out in the Scientific Research Center of Biosafety and Environmental Control in the Agro-Industrial Complex DSAEU.

Study of the biological properties of PEDV was performed using newborn non-immune piglets. Two groups of newborn piglets-analogues ( 6 experimental individuals and 3 control individuals) were randomly selected, before they started to consume colostrum. Piglets of crossbreeds $1 / 2$ Pietren, $1 / 4$ Large White and $1 / 4$ Landras were obtained from a farm free from the PED virus, which was confirmed by preliminary screening studies on presence in the blood, colostrum and feces of both virus and antibodies to PED. Diagnostic analyses of sows' and piglets' samples were performed with RT-PCR and ELISA using commercial test kits "Bio-T kitRPEDV all - TGEV" ("Biosellal", France), "PED IgA ELISA" ("BioNote", Republic of Korea), and "Swinecheck ${ }^{\circledR}$ PED" ("Biovet", Canada).

The emergent characteristics of PEDV were determined in the samples collected in both the newborn non-colostrum piglets-analogous groups.

The piglets were kept in separate specialized insulated plastic containers, with a volume of $1.0 \mathrm{~m}^{3}$. The access to both groups of piglets was operated only through the opening at the top of the containner. The distance from the upper plane of the container to the grill, on which the animals were kept, was not less than $70 \mathrm{~cm}$. The maintenance of each container was carried out by personnel in individual overalls. Every staff person was subjected to additional sanitation just before any manipulation with the piglets. Containers were located about $2 \mathrm{~m}$ apart from each other in a separate vivarium room. In the room, the condi- tions were created to prevent active air movement, which was monitorred by the thermo-anemometer EA-2M (the range of measurement of the air speed within $0.1-5.0 \mathrm{~m} / \mathrm{s}$ ). The air temperature fluctuated in the range of $31-34{ }^{\circ} \mathrm{C}$ during the day and at night $17-23^{\circ} \mathrm{C}$. The conditions of the model experiment were as close as possible to industrial conditions. The experiment was carried out in August in the premises of the DSAEU vivarium without any measures to remove the house flies present - Musca domestica vicina Mcq. (Linnaeus, 1758).

The field strain of the PEDV which was isolated in one of the pig farms in the central region of Ukraine was used as a verified positive sample in the presented model of PED infection. The sequence-analysis showed the relation of this strain to the PED virus that was identified as a North American grouping II from the two group (Masiuk et al., 2017).

The piglets of the experimental group were exposed to the PEDV virus infection. The infection of animals was performed orally in a dose of virions of 1-10 genome equivalents. Control piglets were not infected, but they were kept in the same room as the infected animals. Every piglet before treatment with PEDV was tested for indices of health, especially locomotion and the body temperature. The locomotion of all animals was normal and temperature of the piglets of both the experimental and control groups was in the range $38.0-38.7^{\circ} \mathrm{C}$.

The piglets, that died by reason of diarrhea syndrome due to infection with PEDV, were dissected and the pathanoatomical changes were described. The infectious process was assessed with histological, bacteriological and PCR analyses in the tissue samples taken from the small intestine of the dead animals.

Fragments of the small intestine tissue from the dead piglets were fixed with $4 \%$ formalin solution in saline buffer. The tissue samples were paraffinized and $5 \mu \mathrm{m}$ slices are prepared with a microtome. The slices were dried at room temperature and stained with both hematoxylin and eosin. The histological analysis of the objects was carried out with a microscope Leica DM 1000, digital camera Leica DFC 295, and the software Leica Qwin 3.0.

The samples for PCR analysis were prepared in a glass homogenizer with $100 \mathrm{mg}$ tissue fragments of the small intestine. The extraction of nucleic acids (NA) from the biological material was carried out by the sorption method of these macromolecules on a silica-gel membrane with using columns BioExtract Column (Biosellal, France). The PED was diagnosed by polymerase chain reaction with real-time detection of the results (RT-PCR).

The reverse transcription of the RNA of the PED virus and the replication of the cDNA were performed using a kit Bio-T kit ${ }^{\mathbb{B}}$ PEDV all-TGEV (Biosellal, France) and CFX 96 Real-Time System (Bio Rad, USA) according to the temperature regime (Table 1).

\section{Table 1}

Temperature regime of amplification

\begin{tabular}{cccc}
\hline Phase No. & Temperature, ${ }^{\circ} \mathrm{C}$ & Time, seconds & Number of cycles, pcs. \\
\hline 1 & 50 & 1200 & 1 \\
2 & 95 & 300 & 1 \\
3 & 95 & 10 & 45 \\
4 & 60 & 45 & \\
\hline
\end{tabular}

The fluorescence intensity in the samples was measured in real time at stage 4 of the temperature regime.

The analyses of the amplification results, as well as determination of the threshold cycle, $\mathrm{C}_{\mathrm{t}}$, were carried out using the Bio-Rad CFX Manager software.

Bacteriological study of the microorganism contents in the gastrointestinal tract of the animals was directed on both the indication and identification of microbionts of different taxonomic groups. To isolate aerobic bacteria, simple and enriched nutrient media were used, namely: meat-peptone broth and meat-peptone agar in a native form and with the addition of $10 \%$ blood serum, as well as on cardiac-brain broth, R. Hottinger's media, agar Endo, XLD agar. The anaerobic microorganisms were identified using both the Kit Tarozzi broth and the medium $5 \%$ agar with blood addition.

Morphology and biochemical data of field crops were studied by routine methods. The pathogenicity of the identified daily cultures of microorganisms was determined in a bioassay on white mice. The isola- 
ted culture of microorganisms was administered subcutaneously in a volume $0.3 \mathrm{~cm}^{3} /$ animal to 5 white mice with a live weight of $18-20 \mathrm{~g}$.

Fly individuals in the vivarium were selected to determine PEDV content in both the consumed matter and material absorbed from the body surface of the flies. The samples of PEDV content in the fly organism and absorbed on fly organism surface were studied separately. The flies caught in the vivarium were treated with sterile physiological saline solution to obtain the samples of absorbed PEDV. After individual washing of every fly the solutions were used for PEDV analyses with PCR-RT method. The second group of the flies selected in the vivarium was homogenized after the same washing. These samples were used to determine PEDV as fly consumption path. All samples of "fly consumption path" were randomly divided into two subgroups, which were studied with PCR-RT analysis twice with an interval of 72-hours.

Statistical analyses of the obtained results were carried out with using specialized software Statistica 6 (StatSoft Inc., USA). The reliability of the differences was evaluated after checking the obtained experimental data for the normal distribution using the Student test or its nonparametric analogue, the Wilcoxon test. The sample parameters indicated below in the text have the following notation: $\mathrm{x}$ is the selective mean, SE is the standard error of the mean.

\section{Results}

Non-immune newborn piglets which had not been fed colostrum were randomly selected in a pig enterprise immediately before the start of study. The screening studies of pigs for the presence of PED infection had been previously ( 2 weeks) carried out on the chosen pig farm (Table 2 ).

Table 2

The results of the PED infection screening

in the donor farm of newborn piglets

\begin{tabular}{lccccccc}
\hline \multirow{2}{*}{ The tested groups } & \multicolumn{4}{c}{ ELISA } & \multicolumn{2}{c}{ RT-PCR } \\
\cline { 2 - 7 } & \multicolumn{2}{c}{ serum } & \multicolumn{3}{c}{ colostrum } & \multicolumn{2}{c}{ feces } \\
\cline { 2 - 7 } & $\mathrm{S} / \mathrm{P}, \%$ & result & $\mathrm{S} / \mathrm{P}, \%$ & result & $\mathrm{Ct}$ & result \\
\hline Sows 1-2 farrow & $0.21 \pm 0.04$ & - & $0.47 \pm 0.04$ & - & n.a. & - \\
Sows 3-4 farrows & $0.21 \pm 0.04$ & - & $0.27 \pm 0.07$ & - & n.a. & - \\
Sows 5 farrow and older & $0.21 \pm 0.05$ & - & $0.43 \pm 0.10$ & - & n.a. & - \\
Piglets of 35 days of age & $0.28 \pm 0.04$ & - & n.a. & n.a. & n.a. & - \\
Piglets of 75 days of age & $0.17 \pm 0.05$ & - & n.a. & n.a. & n.a. & - \\
Piglets 150 days old & $0.18 \pm 0.04$ & - & n.a. & n.a. & n.a. & - \\
\hline
\end{tabular}

Note: "-“- negative result; "n.a." - the absence of determined fluorescence.

The results of the screening study showed that the donor farm of the newborn piglets was free of PED infection. This conclusion is confirmed by the negative results of PCR analyses and determination of PEDV specific antibodies in the blood of both the sows and the piglets.

The results obtained in the model of experimental piglet's infection allowed us to assess the characteristics of distinctive temporal features of the growth and the length of the infectious PED process down to $100 \%$ mortality. The observed data showed the first typical features of this disease 18 hours after the induction of infection. One of the six piglets of the experimental group developed primary clinical signs of the disease - muscle weakness, lethargy, common inhibition of motor and reflex activity. In the following two hours, this individual had a manifestation of watery diarrhea, which is the main typical clinical sign of PED. Further clinical indicators of the severity of diarrhea syndrome progressively increased in every piglet of the infected group. The first lethal outcome was detected 60 hours after infection and 48 hours after visual manifestation of the clinical signs of PED.

The incubation period of the next 5 piglets infected with the PEDV developed continued for 6-8 hours longer than in the pig with the earliest symptoms, and was 24-26 hours. The infectious process developed typically in accord with clinical manifestations which fully correspond to the diarrhea syndrome of PED in newborn piglets. The deaths of animals of the experimental group were detected during the 68-72nd hours after infection. Analysis of clinical symptoms showed that every piglet infected with the pathogen had intensive diarrhea signs after an incubation period of the virion of about $22 \pm 4$ hours. The intensity of the pathological process progressively increased right up to the lethal outcome. The observed diarrhea symptoms were accompanied with continuous vomiting in every piglet, and some of them developed extremely painful symptoms. The observed feces immediately liquefied and turned into watery stools, colourless or greenishyellow, without any admixture of blood. The main symptoms of diarrhea were identified in every infected animal. Moreover, diarrhea was prolonged, uninterrupted and quickly led to dehydration. The loss of animal weight was dramatic, which was visually noted, in particular, the piglets had clearly visible ribs and spine. The skin became wrinkled, inelastic, dry, and rough. General deterioration rapidly increased, the piglets noticeably weakened; toxic effects, metabolic acidosis and asthenia increased. During the 68-72nd hours of the development of diarrheal syndrome, on the background of hypothermia and progressive exhaustion, each of the infected pigs died. Thus, the lethality in the group of infected piglets was $100 \%$.

Complex pathognomonic changes in the organism of the infected piglet group presented as the following abnormalities - severe general exhaustion, anemia and signs of severe intoxication combined with metabolic acidosis and alimentary asthenia. The signs of gastroenteritis and desquamation in the small intestine epithelium were meaningful and these features were pronounced in many areas of the gastrointestinal tract. Moreover, the pathogenetic signs of granular dystrophy were observed in the liver, kidneys and the heart muscle. Significant hyperplasia was discovered in the gastrointestinal lymph nodes. Every infected piglet had developed a number of anemic areas in the mucous membranes as well as an attenuation of the vessels, and depletion of the fat depot of the internal organs. In spite of all observed abnormalities, there was no indication of pathology of the spleen. The spleen tissue of the piglets infected with PEDV was not enlarged and the colour was bright-cherry, which reflects an absence of observed damage.

Histological analyses of fixed slices made from the samples of small intestine of both the control group piglets and the animals infected with PEDV indicated mucosal atrophy, swelling and infiltration of the intestinal wall with leukocytes (Fig. 1).

The results of histological analysis showed significant disorder in the structure of the small intestine, especially damage to the superficial and glandular epithelium. These changes are evidence of intestine dystrophy and predict local generation of necrotic areas. Moreover, strongly pronounced desquamation of the epithelial cells in the small intestine (a) accompanied with meaningful swelling (b) of the submucosa were detected in every sample of this piglet group.

Histology staining revealled the fragmentation, decay and vacuolation of muscle cells (Fig. 2a) as well as pyknosis and the translocation of nuclear matter also (Fig. 2b). The analysis of immune cells location showed intensive infiltration of lymphocytes and macrophages into the mucosa and submucosa of microvillies (Fig. 3).

Pathogens of both fungus and yeast microorganisms were not found in the intestinal contents of the orally infected group of piglets. The results of bacteriological analysis of fecal samples and intestinal contents of the experimental piglets infected with PEDV group showed the absence of pathogenic microorganisms (Table 3).

Neither fungus nor yeast microorganism pathogens were found in the intestinal contents of the orally infected group of piglets.

The kinds of elementary transient microflora that are usually are determined as being derived from the environment have been observed in the gullet. Particularly, the following bacteria families were dominant in this study - Enterobacteriaceae, Bacillaceae, Coccaceae. Prokaryotes species were identified according to their morphology and biochemical properties as typical fo newborn piglets. Clostridium perfringens showed an exceptionally high saccharolytic activity as well as anthracoid and coliform bacilli, while vulgar proteium developed extremely high proteolytic activity.

The test of pathogenic capability of isolated prokaryotic samples did not lead to the death of infected white mice for 5 days. Thus, all the identified prokaryotes were nonpathogenic.

Every small intestine sample from the piglets that had died as result of infection was analysed with RT-PCR to determine the PEDV content. The obtained results demonstrated meaningful $C_{t}$ values in infected piglet samples, at average $21.33 \pm 0.68$. The data shown in Figure 4 
indicate that the concentration of the content of the PED virus in piglets whose infection had a lethal outcome corresponds to the quantitative range of $10^{5}-10^{6}$ genomic equivalents of the PEDV virions in $1 \mathrm{~g}$ extracted sample.

It should be noted that the piglets which were not infected with the field virus PEDV developed clinical manifestations of diarrhea syndro- me as well as the infected piglet group. The clinical signs of PED detected in all piglets of the control group were similar to those in the experimental group. However, the manifestation of these symptoms was delayed for several days. The lethal outcome of all piglets of the control group occurred at a more distant period in comparison with the experimental group.

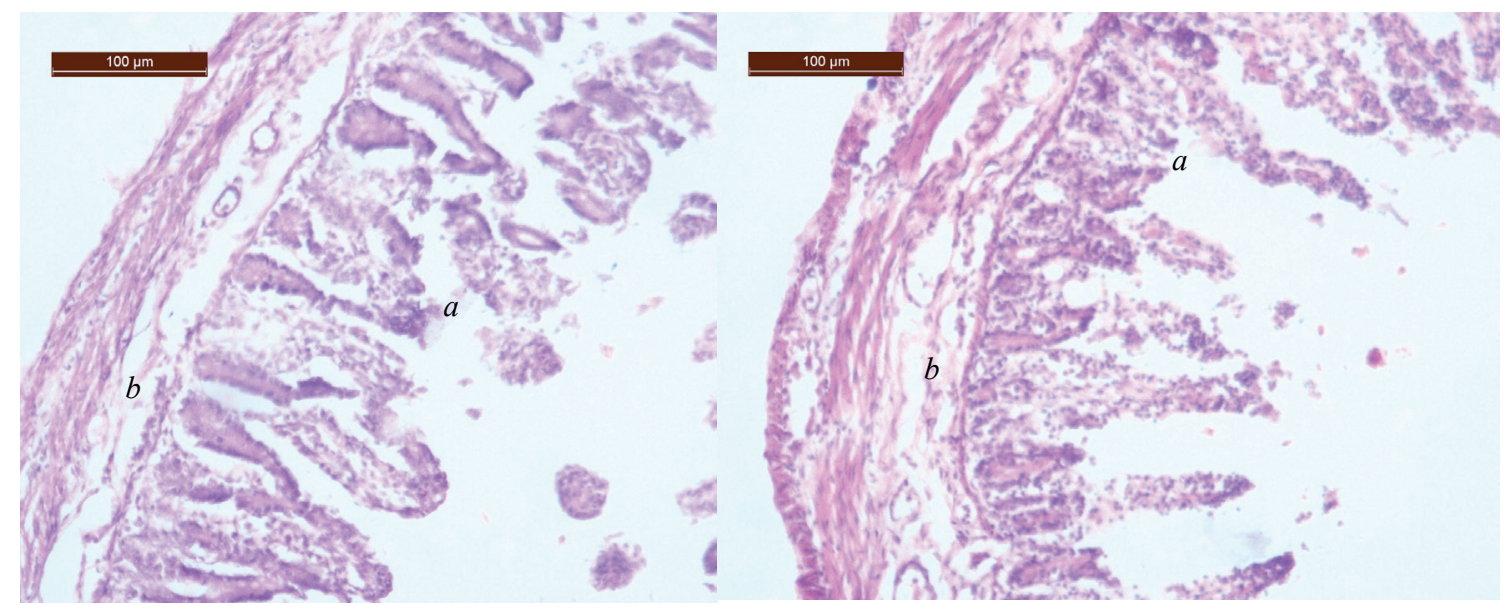

Fig. 1. Results of histology staining of the small intestine of piglet group infected with PEDV (hemotoxylin-eosin staining): $a$-desquamation of small intestinal epithelial cells; $b$ - swelling of the submucosa

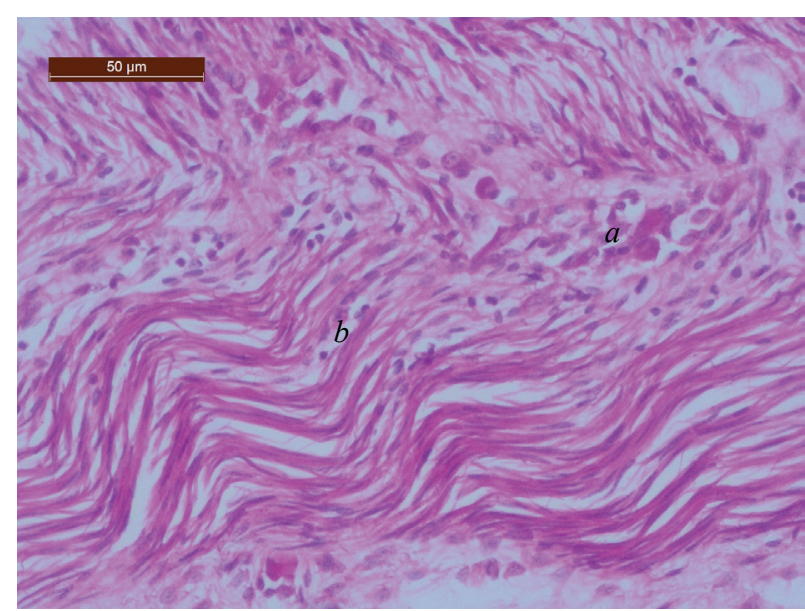

Fig. 2. The fragmentation of muscle in shell of small intestine (hemotoxylin-eosin staining): $a$ - the destruction and vacuolization of myocytes; $b$-the pyknosis and rhexis of myocyte nucleis

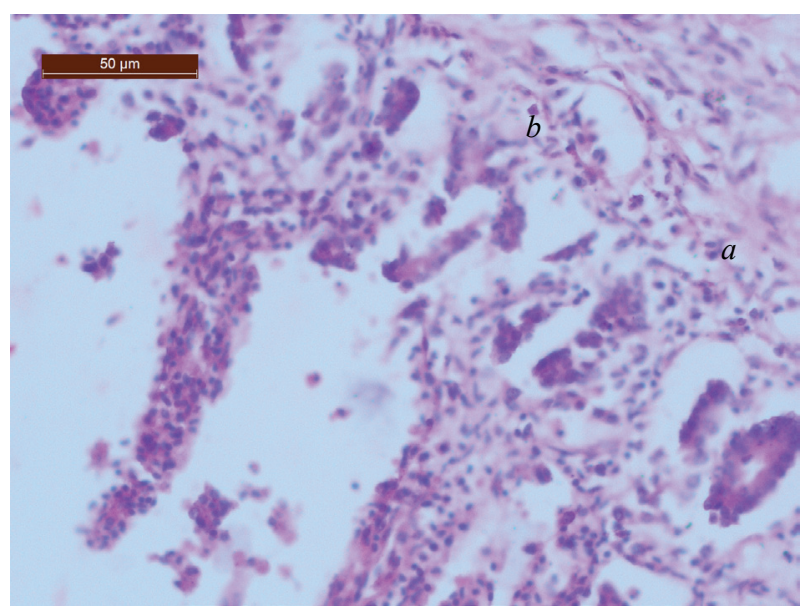

Fig. 3. The mucosa of the small intestine (hemotoxylin-eosin staining): $a$-lymphocytes infiltration; $b$ - macrophage infiltration

Results of the RT-PCR analysis of the small intestine samples from the control group piglets showed a slightly lower PEDV content than the infected group. The $\mathrm{C}_{\mathrm{t}}$ values for the samples obtained in the control group were $23.05 \pm 1.31$, which corresponds to $10^{4}-10^{5}$ genomeequivalents of the virions of the PED exciter in $1 \mathrm{~g}$ of extract.

The first signs of the disease in the piglets of the control group appeared 36 hours after the start of the experiment. By contrast, for the infected group the time elapsing before appearance of the first symptoms did not exceed 18 hours. The result confirms the possibility of the virus spreading within the vivarium from the infected piglets to the piglets of the control group. The temporal differences in the lethal outcome between the groups correspond to the time difference in the manifestation of the first clinical signs.

Table 3

Species composition of the microbiocenosis of the intestines of piglets that died from PED $(n=9)$

\begin{tabular}{|c|c|c|c|c|c|c|c|c|c|}
\hline \multirow{3}{*}{$\begin{array}{c}\text { Name } \\
\text { of microbiont }\end{array}$} & \multicolumn{9}{|c|}{ Number of biomaterial } \\
\hline & \multicolumn{5}{|c|}{ test group } & \multicolumn{4}{|c|}{ control group } \\
\hline & 1 & 2 & 3 & 4 & 5 & 6 & 1 & 2 & 3 \\
\hline $\begin{array}{l}\text { Escherichia coli (Castellani and } \\
\text { Chalmers, 1919) }\end{array}$ & + & + & + & + & + & + & + & + & + \\
\hline Hemolysins $E$. coli & - & - & - & - & - & - & - & - & - \\
\hline Proteus vulgaris (Hauser, 1885) & + & - & - & + & - & + & + & - & + \\
\hline Pseudomonas aeruginosa (Migula, 1900) & + & - & + & + & - & - & + & + & - \\
\hline $\begin{array}{l}\text { Clostridium perfringens (Hauduroy } \\
\text { et al., 1937) }\end{array}$ & - & + & - & + & + & + & - & + & - \\
\hline $\begin{array}{l}\text { Bacillus cereus (Frankland \& } \\
\text { Frankland, 1887) }\end{array}$ & + & - & + & - & + & - & + & + & - \\
\hline Bacillus subtilis (Cohn, 1872) & - & - & - & + & - & + & + & - & + \\
\hline Bacillus mycoides Flügge, 1886 & + & + & - & + & + & - & - & + & + \\
\hline Bacillus megaterium (de Bary, 1884) & - & - & + & - & - & + & + & - & + \\
\hline $\begin{array}{l}\text { Enterococcus spp. (Schleifer \& } \\
\text { Kilpper-Bälz, 1984) }\end{array}$ & + & + & - & + & - & + & - & + & - \\
\hline Staphylococcus spp. (Rosenbach, 1884) & - & + & - & - & + & - & - & + & + \\
\hline $\begin{array}{l}\text { Streptococcus suis (Kilpper-Bälz \& } \\
\text { Schleifer, 1987) }\end{array}$ & - & + & - & - & - & + & + & - & - \\
\hline Klebsiella spp. (Trevisan, 1885) & - & - & - & + & + & - & - & + & - \\
\hline
\end{tabular}

Note: "+" - presence of a microbiont; "-" - absence of microbiont determined content.

The time of the lethal outcome of the infected group was identified as within the $68-72$ nd hours. In the control group, the lethal outcome occurred 88-90 hours after the start of an experimental infection.

In all households, house flies are extremely common (Musca domestica vicina Mcq.), which in the conditions of the experiment (August, 2017) were also present in the vivarium in sufficient quantities. The flies could have been an effective PEDV carrier in these conditions. In favour 
of this assumption is the correspondence between the time from infection till the appearance of clinical symptoms and the temporal difference in the lethal outcome of the infected and control groups. This assumption was confirmed by the results of PCR-RT analysis of the PED virus on the surface and in the body of houseflies. The samples of fly surface were tested on PEDV content. The results of studies of four group samples showed $\mathrm{C}_{\mathrm{t}}=31.44 \pm 0.68$ (Fig. 5), which approximately corresponds to the quantitative range of $10^{2}-10^{3}$ genome-equivalents of the virions of the PED exciter in $1 \mathrm{ml}$ of flushing. The samples of flies were tested on PEDV content too. Two subgroups of individual fly samples were studied with PCR-RT analysis with a 72-hour postponement. The results of the analysis are shown in Figure 6.

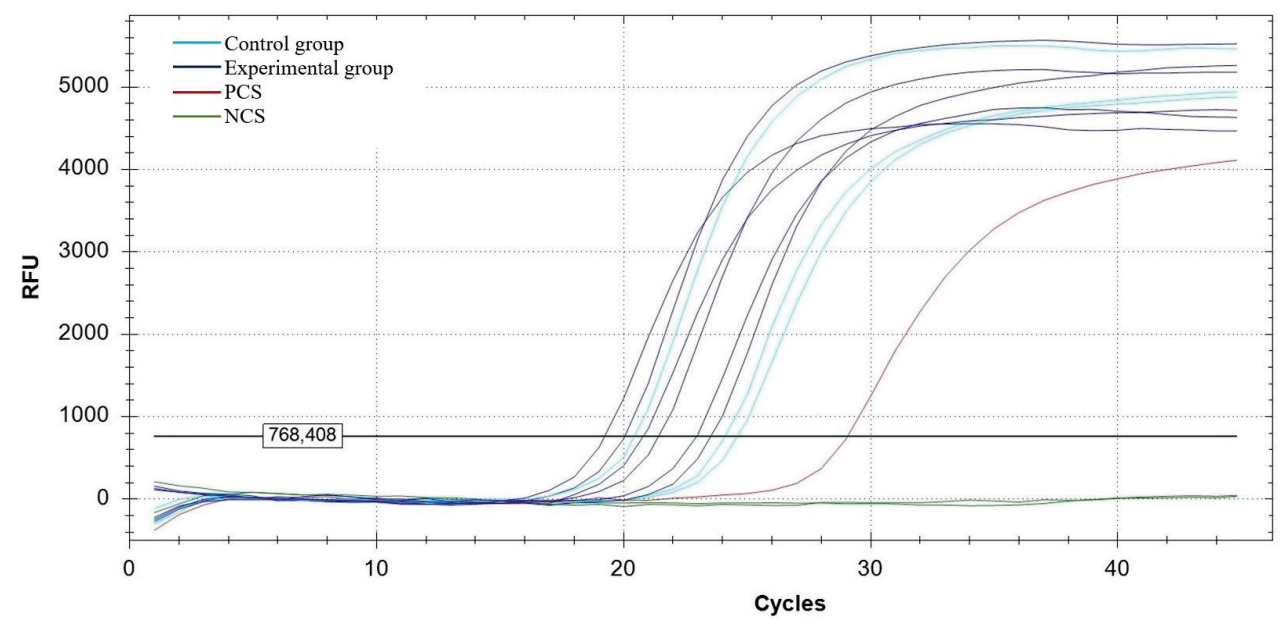

Fig. 4. The kinetics of cDNA amplification products of the PED virus extracted from the small intestine of piglets from the experimental and control groups: NCS - negative control samples RT-PCR, PCS - positive control sample RT-PCR

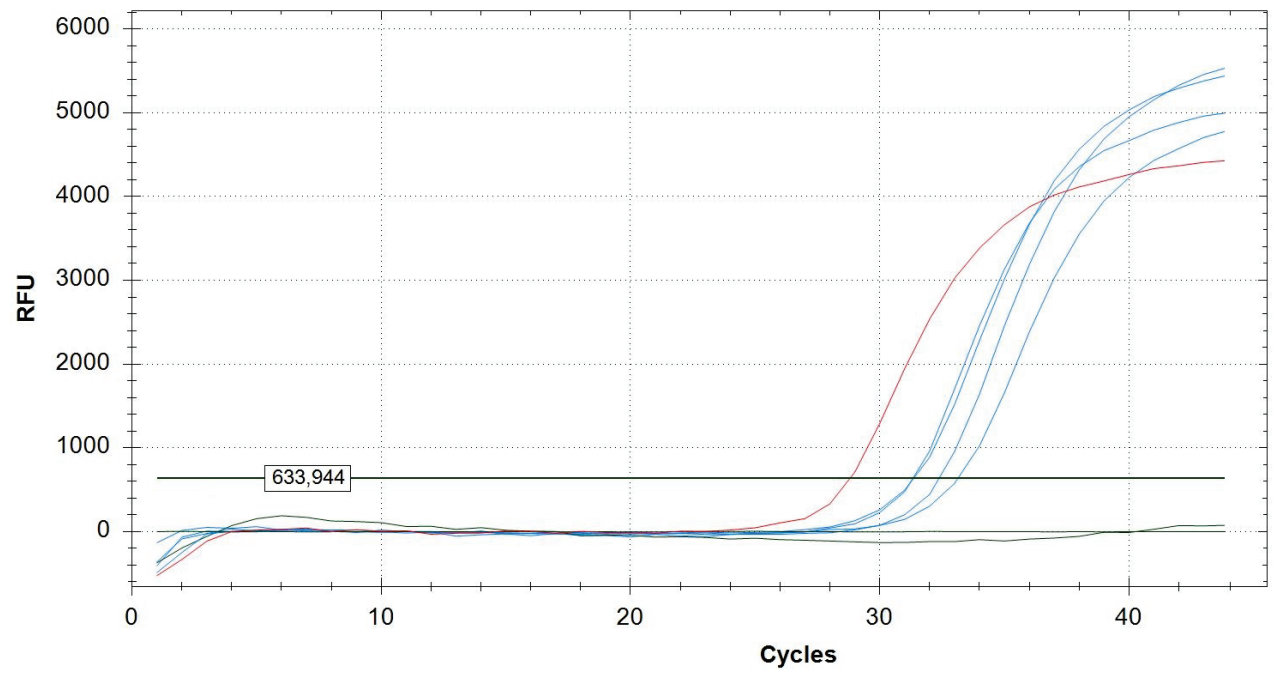

Fig. 5. The kinetics of cDNA amplification products of the PED virus extracted by washing of Musca domestica vicina Mcq.: NCS - negative PCR-RT control samples, PCS - PCR-RT positive control sample

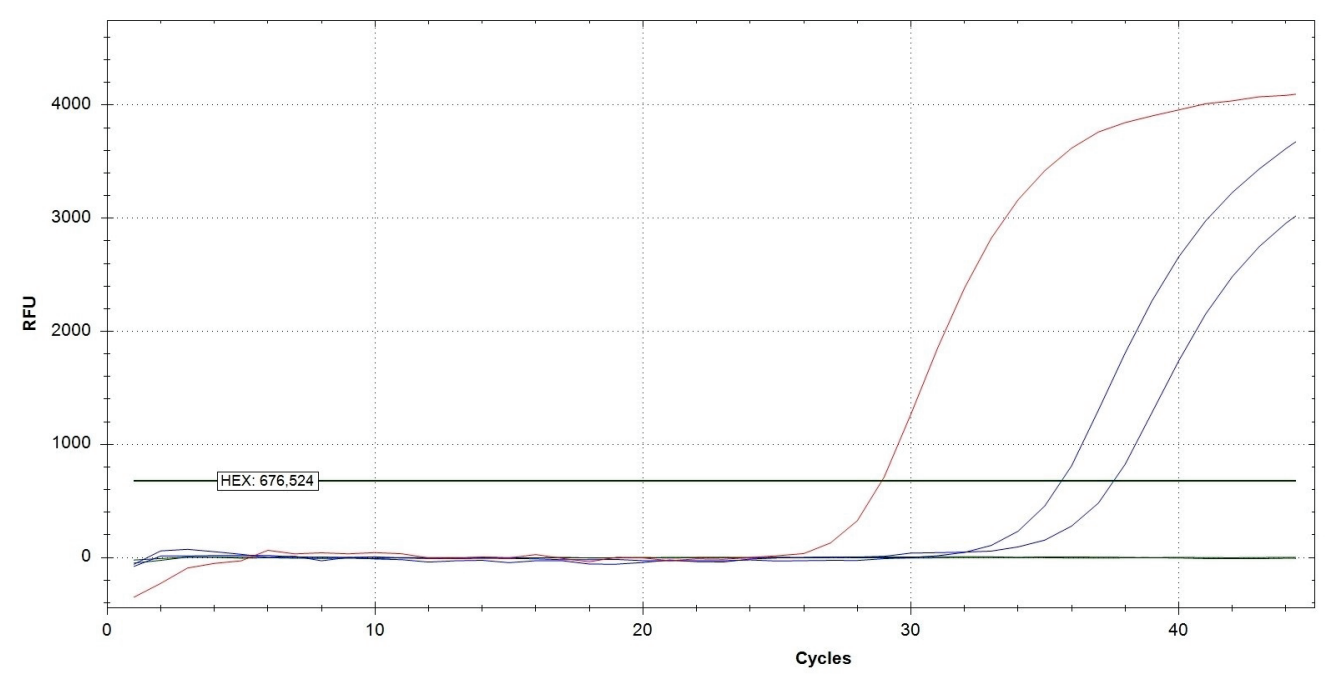

Fig. 6. The kinetics of cDNA amplification products of the PED virus extracted from the homogenate samples of Musca domestica vicina Mcq.: NCS - negative PCR-RT control samples, PCS - PCR-RT positive control sample 
The content of virions revealed in both subgroups was almost identical and the statistical differences were not significant. The $C_{t}$ value determined in the flies homogenate samples selected in the beginning of the experiment was 35.62. In the sample homogenized after 72 hours it was 37.57. The results obtained indicate that reproduction of the PED pathogen in the body of houseflies did not occur.

\section{Discussion}

Infection induced by PEDV was first identified only 5 years ago in Indiana (USA) in May 2013 (Snelson, 2014). Despite the short period since the discovery of PED disease this has become a separate nosological unit of infectious pathology. The risk of significant economic losses in pig farms caused by PED incidents is associated with high mortality of newborn piglets and a decrease in pig farming productivity of older age animal groups. Recently it has been shown that PEDV is an emergent highly pathogenic virus that induces a lethal diarrheal syndrome in newborn piglets (Stevenson et al., 2013). PED infection proceeds as a non-lethal enteral disorder in the cohort of adult animals (Wang et al., 2014; Alvarez et al., 2015; Choudhury et al., 2016).

PEDV differs from other representatives of the Coronaviridae family by its high contagiousness, which is due to the genetic features of this virus (Gong et al., 2017). In the course of one year after the discovery in North America a new variant of the PEDV strain with three deletions, one insertion and several mutations in the $\mathrm{S}$ (spike) 1 region was detected (Wang et al., 2014). Recent results obtained in the largest comparative analyses of 138 PEDV genomes have shown that this virus has a minimum of 6 subtypes (Jang et al., 2018). Groups 1 and 2 come from North America and groups 3-5 from Asia. According to Jang et al. (2018), the rate of the genome evolution is $3.38 \times 10^{-4}$ site replacements per year, which is similar to other RNA-containing viruses.

In this way, the relatively high mutagenic ability of PEDV determines the difficulties of developing effective vaccines against PEDV. The lack of immunity in newborn piglets allows the virus to induce large-scale infection of piglets in a short period.

The spread of PEDV is also promoted by the fact that the virus can remain viable in the external environment for up to six months. At the same time, in the convalescent organism no viruses were revealed (Goede \& Morrison, 2016). The high contagiousness and pathogenicity of PEDV along with significant economic losses caused by PEDV infection are the main causes of the special attention and intensive study of PED infection around the world. However, despite the progressive growth in the number of publications on the subject, the most important key biological characteristics of this pathogen remain too insufficiently studied to develop an effective strategy to cure PEDV infection.

The results of our study of the virulent activity characteristics of PEDV field isolated in Ukraine showed that the minimal infecting dose is $1-10$ virions, which is equivalent to Dosis Certa Letalis (DCL). These data indicate that the PEDV strain isolated in Ukraine is an emergent highly virulent pathogen for newborn piglets. The results obtained in our work demonstrate that viral isolate induces infectious pathology in piglets according to the classic type of epizootic process, which is lethal for the newborns. Therefore, they act as a biological indicator of PEDV circulation in the focus of infection (Masiuk et al., 2017a).

High virulence of the PED pathogen has been shown in the realized experimental model, which contributes to the active spread of the virus to the nearest areas by mechanical transmission through transport, inventory, contaminated feed, insects, etc. (Yeruham et al., 1996; Sven et al., 2007; Förster et al., 2009).

Particular importance in the epidemiology of infectious diseases is given to flies as a separate biological carrier of microorganisms in animal populations, as well as translocation between animals and humans (Lecuona et al., 2005; Sven et al., 2007; Sukontason et al., 2007; Holt et al., 2007; Barin et al., 2010; Gestmann et al., 2012).

The biological features of $M$. domestica should be noted, which are important in providing an epizootic process of infectious diseases. The results presented by Dübendorfer et al. (2002) showed that the house fly M. domestica vicina Mcq. is a dipterous insect of the family of real flies, a common synanthropic organism, which almost does not occur in the wild environment. It is a diurnal insect. It feeds on organic waste of animals, but develops in their feces during the larval period, which contributes to their role as a mechanical carrier of infectious and parasitic pathogens. It has been proved that $M$. domestica is capable of transmitting several microorganisms, especially enterotoxic E. coli, Klebsiella, Campylobacter, Providencia, Staphylococcus aureus, Streptococcus viridans, etc. (Förster et al., 2012).

The epidemiological potential of $M$. domestica related to the mechanical propagation of the agents of pig infectious diseases is well known. The fly can spread various infectious agents, especially Aujeszky's disease (Medveczky et al., 1988), transmissible gastroenteritis (Saif \& Wesley, 1999), mycobacteriosis (Fischer et al., 2001), reproductiverespiratory syndrome of pigs (Otake et al., 2004), as well as several parasitic diseases - Ascaris suum, Strongyloides ransomi, Metastrongylus sp., undetermined Strongylida is recorded (Förster et al., 2009).

Based on the results obtained in a study of the biological properties of $M$. domestica, Murvosh \& Taggard (1966) identified two main propagation mechanisms of microorganisms which are mediated by this insect species. The first of them is the mechanical transfer of pathogens on the surface of insect bodies due to the adsorption of microorganisms and viruses on numbered setae and filaments. The second is carried out as a result of regurgitation of incompletely digested food which is contaminated with infectious agents.

In the world scientific literature there have been no reports that M. domestica may be a vector for the spread of PEDV. The results obtained in our study confirm the possibility of mechanical spreading of the PED virus transferred on the surface of the body of $M$. domestica. This is evidenced by the detection with the RT-PCR the genetic material of PEDV in the samples washed from the surface of the bodies of flies which had been in contact with the infected piglets.

Results of the study of homogenates from $M$. domestica bodies prepared at an interval of 72-hours with RT-PCR showed no PEDV replication in the flies. This is confirmed by the unchanged $C_{t}$ value in the samples isolated from flies 3 days after the beginning of infection of the piglets. The results presented in our study indicate that flies can dominate only as a vector of mechanical transferring of PEDV.

The measurement of fly activity in the environment show that M. domestica has an average speed of $6.4 \mathrm{~km} / \mathrm{h}$ and flight distance can be up to $40 \mathrm{~km}$ (Golding et al., 2001). The summer activity of flies could form one of the main factors of total environmental contamination with PEDV in a relatively short time period. Taking into account that newborn piglets have the lack of maternal immunity and that their own immune system is immature, they are extremely sensitive to PEDV infection propagated by flies. Generation of infection in piglets poses a serious threat to the spread of PEDV not only inside a farm, but it could be a source of infection in pigs in the farms located within a radius of several dozens of kilometers from the primary infected area.

According to data analysis of PubMed, ScienceDirect and Google Scholar publications, the positive results of infection of piglets by transmission of PEDV by house flies were obtained for the first time. The fact of mechanical spread of PEDV by house flies which contributes to the infection of piglets is experimentally established. The results of the study of the emergent properties of PEDV are fully consistent with the literature data on of the development of the PED disease. In the presented experimental model (summer, 2017) of acute intestinal disorder in the newborn piglets, typical features of PEDV infection developed within 36-48 hours after the injection of the strain of the virus isolated in Ukraine strain. The dosed infection of newborn piglets resulted in $100 \%$ lethal outcome. This fact is an important feature of the epizootic process of PED. The obtained results indicate that this manner of spreading of the virus could be one of the leading paths of infection on Ukrainian farms and a significant critical factor in the epidemiology of PED. These results have a great practical importance and can be used to develop a preventive strategy and to specify the therapy of PED infection in real pig farming conditions.

The presented results of the research on the emergent characters of PEDV infection point to the relevance of the problem of the viruses spreading on Ukrainian farms. The process of PEDV propagation on Ukrainian farms requires further detailed study, especially the pathoge- 
nic proprieties of field isolated strains. The most significant issue is to determine the relation of the biological properties of PEDV field isolates with their genetic characteristics. First of all, biological properties of PEDV relate to the cause-effect relationship between the pathogenicity of the virus, the nucleotide sequence of its genome and the epizootic features of the infectious pathology. The differences among all PEDV morbid events could be caused by various isolates of the pathogen which are circulating among the susceptible population. Thus PED infection in Ukraine requires urgent and widespread study.

The extremely limited data on the characteristics of the infectious process of PED in Ukraine compared with other countries indicates that further research should be conducted on the pathways and methods of PEDV spreading on farms. The features of the spread of PEDV in the environment, the duration of preservation of the viruses' virulent properties in the conditions of virions' adsorption on contaminated technological equipment and also in the organism of both susceptible and nonsusceptible animals remain poorly studied.

\section{Conclusions}

In the model of PED piglets' infection it is established that the tested PEDV strain has a high virulence for newborn piglets, DCL is 110 virions and the incubation period is $18-26$ hours. PED is acute with lethality to $100 \%$ within $68-72$ hours after infection.

Based on the results of RT-PCR analyses in the samples washed from the surface of the bodies of flies, it is established that one of the ways piglets are infected and the environment contaminated with the PED virus is the spread of the pathogen by the flies $M$. domestica vicina Mcq. This leads to the induction of the emergent form of PED in piglets.

The presence of PEDV in the homogenate from $M$. domestica vicina Mcq. bodies caught in the focus of infection and the absence of virus reproduction in their body confirms the role of housefly in the mechanical spread of PEDV in the external environment.

\section{References}

Alvarez, J., Sarradell, J., Morrison, R., \& Perez, A. (2015). Impact of porcine epidemic diarrhea on performance of growing pigs. PLoS One, 10(3), e0120532.

Annamalai, T., Saif, L. J., Lu, Z., \& Jung, K. (2015). Age-dependent variation in innate immune responses to Porcine epidemic diarrhea virus infection in suckling versus weaned pigs. Veterinary Immunology and Immunopathology, 168, 193-202.

Arriba, M. L., Carvajal, A., Pozo, J., \& Rubio, P. (2002). Isotype-specific antibodysecreting cells in systemic and mucosal associated lymphoid tissues and antibody responses in serum of conventional pigs inoculated with PEDV. Veterinary Immunology and Immunopathology, 84(1), 1-16.

Barin, A., Arabkhazaeli, F., Rahbari, S., \& Madani, S. A. (2010). The housefly, Musca domestica, as a possible mechanical vector of Newcastle disease virusin the laboratory and field. Medical and Veterinary Entomology, 24, 88-90.

Boyko, A., Brygadyrenko, V., Shendryk, L., \& Loza, I. (2009). Estimation of the role of antropo-zoonosis invasion agents in the counteraction to bioterrorism. Counteraction to Chemical and Biological Terrorism in East European Countries. NATO Science for Peace and Security Series A: Chemistry and Biology. Springer Nature, 309-315.

Carvajal, A., Argüello, H., Martínez-Lobo, F. J., Costillas, S., Miranda, R., de Nova, P. J. G., \& Rubio, P. (2015). Porcine epidemic diarrhoea: New insights into an old disease. Porcine Health Management, 1(12), 1-8.

Choudhury, B., Dastjerdi, A., Doyle, N., Frossard, J. P., \& Steinbach, F. (2016) From the field to the lab - An European view on the global spread of PEDV. Virus Research, 226, 40-49.

Dastjerdi, A., Carr, J., Ellis, R. J., Steinbach, F., \& Williamson, S. (2015). Porcine epidemic diarrhea virus among farmed pigs, Ukraine. Emerging Infectious Diseases, 21(12), 2235-2237.

Diel, D. G., Lawson, S., Okda, F., Singrey, A., Clement, T., Fernandes, M. H. V., Christopher-Hennings, J., \& Nelson, E. A. (2016). Porcine epidemic diarrhea virus: An overview of current virological and serological diagnostic methods. Virus Research, 226, 60-70.

Dübendorfer, A., Hediger, M., Burghardt, G., \& Bopp, D. (2002). Musca domestica a window on the evolution of sex-determining mechanisms in insects. International Journal of Developmental Biology, 46(1), 75-79.

Fischer, O., Mátlová, L., Dvorská, L., Švástová, P., Bartl, J., Melichárek, I., Weston, R. T., \& Pavlík, I. (2001). Diptera as vectors of mycobacterial infections in cattle and pigs. Journal Medical and Veterinary Entomology, 15(2), 208-211.
Förster, M., Gestmann, F., Mehlhorn, H., Sievert, K., Messler, S., Neuhausen, N., Petersdorf, S., \& Pfeffer, K. (2012). Flies as vectors of microorganisms potentially inducing severe diseases in humans and animals. Arthropods as Vectors of Emerging Diseases. Parasitology Research Monographs. Vol. 3. Springer, Berlin, Heidelberg.

Gerber, P. F., Gong, Q., Huang, Y. W., Wang, C., Holtkamp, D., \& Opriessnig, T. (2014). Detection of antibodies against porcine epidemic diarrhea virus in serum and colostrum by indirect ELISA. The Veterinary Joumal, 202(1), 3-36.

Goede, D., \& Morrison, R. B. (2016). Production impact and time to stability in sow herds infected with Porcine epidemic diarrhea virus (PEDV). Preventive Veterinary Medicine, 123(1), 202-207.

Golding, Y. C., Roland Ennos, A., \& Edmunds, M. (2001). Similarity in flight behaviour between the honeybee, Apis mellifera (Hymenoptera: Apidae) and its presumed mimic, the dronefly Eristalis tenax (Diptera: Syrphidae). Journal Experimental Biology, 204(1), 139-145.

Gong, L., J. Li, Q., Zhou, Z., Xu, L., Chen, Y., Zhang, C., Xue, Z., Wen, Y. C. (2017). A New Bat-HKU2-like coronavirus in swine, China, 2017. Emerging Infectious Diseases, 23(9), 1607-1609.

Holt, P. S., Geden, C. J., Moore, R. W., \& Gast, R. K. (2007). Isolation of Salmonella enterica serovar enteritidis from houseflies (Musca domestica) found in rooms containing Salmonella serovar enteritidis-challenged hens. Applied and Environmental Microbiology, 73(19), 6030-6035.

Jang, J., Yoon, S. H., Lee, W., Yu, J., Yoon, J., Shim, S., \& Kim, H. (2018). Timecalibrated phylogenomics of the Porcine epidemic diarrhea virus: Genomewide insights into the spatio-temporal dynamics. Genes Genomics, 40(8), 825-834.

Kim, O., \& Chae, C. (2003). Experimental infection of piglets with a Korean strain of Porcine epidemic diarrhoea virus. Journal of Comparative Pathology, 129(1), 55-60.

Langel, S. N., Paim, F. C., Lager, K. M., Vlasova, A. N., \& Saif, L. J. (2016). Lactogenic immunity and vaccines for Porcine epidemic diarrhea virus (PEDV): Historical and current concepts. Virus Research, 226, 93-107.

Lecuona, R. E., Turica, M., Tarocco, F., \& Crespo, D. C. (2005). Microbial control of Musca domestica (Diptera: Muscidae) with selected strains of Beauveria bassiana. Journal of Medical Entomology, 42(3), 332-336.

Lee, C. (2015). Porcine epidemic diarrhea virus: An emerging and re-emerging epizootic swine virus. Virology Journal, 12, 193.

Martelli, P., Lavazza, A., Nigrelli, A. D., Merialdi, G., Alborali, L. G., Pensaert, M. B. (2008). Epidemic of diarrhoea caused by Porcine epidemic diarrhoea virus in Italy. Veterinary Record, 162(10), 307.

Masiuk, D. M., Sosnitsky, O. I., Nedzvetsky, V. S., Kokarev, A. V., \& Koliada, S. G. (2017a). Endemic course of epidemic diarrhea of pigs in the stabilized focus of infection. Regulatory Mechanisms in Biosystems, 8(3), 410-416.

Masiuk, D. M., Sosnitsky, O. I., Nedzvetsky, V. S., Kokarev, A. V., \& Koliada, S. G. (2017b). Epidemiology, etiology and gene analysis of spike $S$ protein of porcine epidemic diarrhea virus infection in Ukraine during 2016-2017. Regulatory Mechanisms in Biosystems, 8(4), 602-610.

Medveczky, I., Kovács, L., Kovács, F. Sz., \& Papp, L. (1988). The role of the housefly, Musca domestica, in the spread of Aujeszky's disease (pseudorabies). Journal Medical and Veterinary Entomology, 2(1), 81-86.

Murvosh, C. M., \& Taggard, C. W. (1966). Ecological studies of the housefly. Annals of the Entomological Society of America, 59, 534-547.

Otake, S., Dee, S. A., Moon, R. D., Rossow, K. D., Trincado, C., \& Pijoan, C. (2004). Studies on the carriage and transmission of porcine reproductive and respiratory syndrome virus by individual houseflies (Musca domestica). Veterinary Record, 154(3), 80-85.

Puranaveja, S., Poolperm, P., Lertwatchharasarakul, P., Kesdaengsakonwut, S., Boonsoongnem, A., Urairong, K., Kitikoon, P., Choojai, P., Kedkovid, R., Teankum, K., \& Thanawongnuwech, R. (2009.). Chinese-like strain of Porcine epidemic diarrhea virus, Thailand. Emerging Infectious Diseases, 15, 1112-1115.

Saif, L. J., \& Wesley, R. D. (1999). Transmissible gastroenteritis and porcine respiratory coronavirus, in straw. Diseases of Swine. 8-th ed. Iowa State University Press, Ames. Pp. 295-326.

Song, D. S., Oh, J. S., Kang, B. K., Yang, J. S., Song, J. Y., Moon, H. J., Kim, T. Y., Yoo, H. S., Jang, Y. S., \& Park, B. K. (2005). Fecal shedding of a highly cellculture-adaptrd porcine epidemic diarrhea virus after oral inoculation in pigs. Journal of Swine Health and Production, 13(5), 269-272.

Srijangwad, A., Stott, C. J., Temeeyasen, G., Senasuthum, R., Chongcharoen, W., Tantituvanont, A., \& Nilubol, D. (2017). Immune response of gilts to single and double infection with Porcine epidemic diarrhea virus. Archives of Virology, 162, 2029-2034.

Stevenson, G. W., Hoang, H., Schwartz, K. J., Burrough, E. R., Sun, D., Madson, D., Cooper, V. L., Pillatzki, A., Gauger, P., Schmitt, B. J., Koster, L. G., Killian, M. L., \& Yoon, K. J. (2013). Emergence of Porcine epidemic diarrhea virus in the United States: Clinical signs, lesions and viral genomic sequences. Journal of Veterinary Diagnostic Investigation, 25, 649-654. 
Strizhakova, O. M. (2013). Isolation and identification of epizootic diarrhea virus in pigs under outbreak at a large farm. Sel'skokhozyaistvennaya Biologiya, 4, 65-69.

Sukontason, K. L., Bunchoo, M., Khantawa, B., Piangjai, S., Rongsriyam, Y., \& Sukontason, K. (2007). Comparison between Musca domestica and Chrysomya megacephala as carriers of bacteria in Northern Thailand. Journal of Tropical Medicine and Public Health, 38(1), 38-44.

Sven, F. M., Heinz, K. M., Kai, S. S., \& Pfeffer, M. K. (2007). Pilot study on synanthropic flies (e.g. Musca, Sarcophaga, Calliphora, Fannia, Lucilia, Stomoxys) as vectors of pathogenic microorganisms. Parasitology Research, 101(1), 243-246.
Tun, H. M., Cai, Z., \& Khafipour, E. (2016). Monitoring survivability and infectivity of Porcine epidemic diarrhea virus (PEDV) in the infected on-farm Earthen manure storages (EMS). Frontiers in Microbiology, 7, 265-276.

Vlasova, A. N., Marthaler, D., Wang, Q., Culhane, M. R., Rossow, K. D., Rovira, A., Collins, J., \& Saif, L. J. (2014). Distinct characteristics and complex evolution of PEDV strains, North America, May 2013 - February 2014. Emerging Infectious Diseases, 20(10), 1620-1628.

Wang, L., Byrum, B., \& Zhang, Y. (2014). New variant of Porcine epidemic diarrhea virus, United States. Emerging Infectious Diseases, 20(5), 917-919.

Yeruham, I., Braverman, Y., Shpigel, N. Y., Chizov-Ginzburg, A., Saran, A., \& Winkler, M. (1996). Mastitis in dairy cattle caused by Corynebacterium pseudotuberculosis and the feasibility of transmission by houseflies. The Veterinary Quarterly, 18(3), 87-89. 